\title{
Management and prevention of obesity in adults and children
}

\author{
Sarah D. McDonald
}

$\infty$

See related article page IIo3

$\mathrm{A}$ s noted in the 2006 Canadian Clinical Practice Guidelines on the Management and Prevention of Obesity in Adults and Children, included as a supplement to this issue of CMAJ (the full document is available online at www.cmaj.ca/cgi/content/full//r6/8/SI/DCI), ${ }^{1}$ approximately $\mathrm{I}$ in Io Canadian children and $\mathrm{I}$ in 4 adults are already obese (defined as weight greater than the 95th percentile for children and body mass index [BMI] greater than $30 \mathrm{~kg} / \mathrm{m}^{2}$ for adults). However, with an additional quarter of children and more than half of adults classified as overweight, rates of obesity are projected to soar.

The guidelines include both a review of the current literature as well as highlighted areas for further research in each of the chapters. Caregivers will also find practical summaries of recommendations to guide care. Unlike most other obesity guidelines, this initiative was produced by a multidisciplinary group of health professionals that included physicians, psychologists, registered dietitians and bariatric surgeons who attempted to address all aspects of the prevention and management of obesity in children and adults. As a consequence, the guidelines address diet, exercise, the environment, pharmacotherapy, behavioural therapy and surgery. The scope of the work and the effort from the many contributors have resulted in a commendable document.

The evidence-based approach used to develop the guidelines is described in chapter I of the full document. As part of the methods for data acquisition and literature review, 4 databases (MEDLINE, EMBASE, Cochrane Controlled Clinical Trials Register and HealthSTAR) were to be searched systematically to identify all relevant studies (in English and nonEnglish) that had an English abstract. Although this approach is ideal, in some chapters only MEDLINE was searched, and in others no search strategy was described. Unlike some other guidelines, ${ }^{2}$ most chapters did not provide the number of studies that met the inclusion criteria and hence were used to formulating the recommendations. The authors did, however, adopt consistent and easy wording to convey the strength of each recommendation, using "we recommend" for grade A (strong) recommendations and "we suggest" for grade B (intermediate) ones, and they assigned a level (from I to 4) to each recommendation to indicate the quality of evidence upon which it was based.

Following the outline of the methods used to develop the guidelines is a review of the classification of obesity and a detailed examination of treatment options. The guidelines draw attention to the importance of waist circumference, a measure of abdominal fat, as an independent predictor of clinically important outcomes. Along with weight and BMI, waist cir- cumference now forms the third "vital sign" of obesity. ${ }^{3}$ In terms of treatment strategies, various conventional methods of weight loss, including diet, exercise, pharmacotherapy and behavioural therapy, are evaluated. Combined therapies are thought to be moderately more effective than single methods. Weight loss of $0.5^{-I} \mathrm{~kg}(\mathrm{I}-2 \mathrm{lb})$ per week for 6 months is sustainable in many patients. ${ }^{2}$ As pointed out in the chapter on pharmacotherapy, it would be important to stress that even modest weight loss in the range of $5 \%-10 \%$ of body weight has been shown to improve outcomes such as blood pressure and lipid and glucose levels. Once the weight has been lost, the guidelines note that the focus must shift to long-term

\section{Along with weight and body mass index, waist cir- cumference now forms the third "vital sign" of obesity.}

weight maintenance, for which exercise has proven to be advantageous. Pharmacotherapy may also prove modestly beneficial, although sibutramine (Meridia, Reductil) and orlistat (Xenical) have recognized cardiovascular and gastrointestinal side effects, respectively, and the long-term safety and efficacy is unknown.

To date, the conventional treatments, either alone or in combination, have not yielded the substantial weight loss reported with bariatric surgery. The Swedish Obese Subjects Study, the landmark study in this field, reported a decrease in weight of I6.I\% at Io-year follow-up among participants who underwent gastric surgery, as compared with a r.6\% increase in weight among contemporaneously matched, conventionally treated controls. ${ }^{4}$ Following the surgical intervention, the authors observed a reversal of many associated comorbid conditions such as diabetes, sleep apnea and hypertriglyceridemia. However, a number of concerns regarding bariatric surgery remain. First, the only large long-term follow-up study, the Swedish Obese Subjects Study, ${ }^{4}$ was not randomized, which may mean there was selection bias. Second, longterm weight loss beyond ro years is unknown and, more importantly, there are no data regarding long-term conse- 
quences of bariatric surgery on clinically important outcomes such as overall mortality. Third, the rate of postoperative complications, including death, was lower than typically reported in clinical reports from many centres. Therefore, postoperative complications under usual-care conditions may be much higher than rates reported in the Swedish Obese Subjects Study. The National Institutes of Health guidelines recommend that only patients with a BMI greater than $40 \mathrm{~kg} / \mathrm{m}^{2}$, or greater than $35 \mathrm{~kg} / \mathrm{m}^{2}$ with high-risk comorbidities, be considered as surgical candidates. ${ }^{5}$

The lack of effective preventive measures and adequate treatment options for obesity that result in substantial weight loss (apart from surgery) is in part due to the lack of large long-term studies in the literature. For instance, I8 randomized controlled trials of interventions for treating obesity in children have been published, but there were fewer than 1000 participants in total. Given the small number of patients in obesity treatment trials, few inferences may be drawn. ${ }^{6}$ Most studies involving both children and adults have had short follow-up periods, which compounds the problem. Through to at least 2008 , it is expected that obesity will continue to be the focus of the Institute for Nutrition, Metabolism and Diabetes of the Canadian Institutes of Health Research, and the accompanying funding should, I hope, begin to address gaps in our knowledge.

An important omission in the 2006 obesity guidelines is the lack of any mention of screening for smoking, despite a recognized association with both obesity and mental health disorders (to which a chapter is devoted). Also, there is little mention of pregnancy, even though obesity increases the risk of infertility and adverse pregnancy outcomes (e.g., preeclampsia, gestational diabetes, cesarean section and wound infection). In addition, maternal obesity is a risk factor for infant macrosomia, and each I-kg increase in birth weight has been found to increase the risk of overweight in adolescence by $30 \%-50 \% .^{7}$ Recently, the liberal recommendations of the US Institute of Medicine for gestational weight gain ${ }^{8}$ have been questioned, ${ }^{9}$ because an increase of more than $25 \mathrm{lb}$ (II.4 kg) was found to have little impact on improving smallfor-gestational-age birth weights but did contribute to maternal postpartum obesity. ${ }^{10}$

The 2006 obesity guidelines are timely. Health care practitioners and members of the public are seeking high-quality information on the prevention and management of obesity in adults and children, as evidenced by the fact that 3 of the 20 most frequently accessed Cochrane reviews in October 2006 were related to obesity. ${ }^{11}$ Unlike a systematic review, whose sole function is to summarize the best evidence available at a given time, the 2006 obesity guidelines have a much larger goal: to effect change. They will be judged successful by the extent to which they change practice, stimulate appropriate research and, ultimately, decrease the prevalence of obesity and its complications among Canadian children and adults. I look forward to seeing the impact of this extensive body of work.

\section{This article has been peer reviewed.}

Sarah McDonald is with the Department of Obstetrics and Gynecology, McMaster University, Hamilton, Ont.

Competing interests: None declared.

\section{REFERENCES}

I. Lau DCW, Douketis JD, Morrison K, et al; Obesity Canada Clinical Practice Guidelines Expert Panel. 2006 Canadian clinical practice guidelines on the management and prevention of obesity in adults and children [summary]. CMAJ 2007;176(8 Suppl):SI-I3. [For the complete set of guidelines, go to www.cmaj.ca/cgi/content /full/I76/8/SI/DCI]

2. Clinical guidelines on the identification, evaluation, and treatment of overweight and obesity in adults - the evidence report. National Institutes of Health. Obes Res i998;6(Suppl 2):5IS-209S.

3. Rippe JM, Crossley S, Ringer R. Obesity as a chronic disease: modern medical and lifestyle management. JAm Diet Assoc 1998;98:S9-I5.

4. Sjostrom L, Lindroos AK, Peltonen M, et al. Lifestyle, diabetes, and cardiovascular risk factors io years after bariatric surgery. N Engl J Med 2004;35I:2683-93.

5. Gastrointestinal surgery for severe obesity: National Institutes of Health Consensus Development Conference Statement. Am J Clin Nutr 1992;55(Suppl 2):615S-9S.

6. Summerbell CD, Ashton V, Campbell KJ, et al. Interventions for treating obesity in children. Cochrane Database Syst Rev 2003; (3):CDoor872.

7. Gillman MW, Rifas-Shiman S, Berkey CS, et al. Maternal gestational diabetes, birth weight, and adolescent obesity. Pediatrics 2003;III:e22I-6.

8. Committee on Nutritional Status During Pregnancy and Lactation, Institute of Medicine. Nutrition during pregnancy. Washington (DC): National Academy Press; 1990.

9. Feig DS, Naylor CD. Eating for two: Are guidelines for weight gain during pregnancy too liberal? Lancet I998;351:1054-5.

Io. Keppel KG, Taffel SM. Pregnancy-related weight gain and retention: implications of the I99o Institute of Medicine guidelines. Am J Public Health 1993;83:1100-3.

II. Top 20 reviews accessed from The Cochrane Library, October 2006. Cochrane News 2006;(38):I4. Available: www.cochrane.org/newslett/CochraneNews 38 lowrescorrected.pdf (accessed 2007 Mar 7).

Correspondence to: Dr. Sarah D. McDonald, Department of Obstetrics and Gynecology, McMaster University, I200 Main St. W, Rm. HSC $3 N_{52}$, Hamilton ON L8N 3Z5; mcdonals@mcmaster.ca 